\title{
SOME PECULIAR MINIMAL SITUATIONS BY FINITE $p$-GROUPS
}

\author{
ZVONIMIR JANKO \\ University of Heidelberg, Germany
}

\begin{abstract}
In this paper we show that a finite $p$-group which possesses non-normal subgroups and such that any two non-normal subgroups of the same order are conjugate must be isomorphic to $M_{p^{n}}=\langle a, b| a^{p^{n-1}}=$ $\left.b^{p}=1, n \geq 3, a^{b}=a^{1+p^{n-2}}\right\rangle$, where in case $p=2$ we must have $n \geq 4$. This solves Problem Nr. 1261 stated by Y. Berkovich in [1]. In a similar way we solve Problem Nr. 1582 from [1] by showing that $M_{p^{n}}$ is the only finite $p$-group with exactly one conjugate class of non-normal cyclic subgroups.

Then we determine up to isomorphism all finite $p$-groups which possess non-normal subgroups and such that the normal closure $H^{G}$ of each nonnormal subgroup $H$ of $G$ is the largest possible, i.e., $\left|G: H^{G}\right|=p$. It turns out that $G$ is either the nonabelian group of order $p^{3}, p>2$, and exponent $p$ or $G$ is metacyclic. This solves the Problem Nr. 1164 stated by Berkovich [1].

We classify also finite 2-groups with exactly two conjugate classes of four-subgroups. As a result, we get three classes of such 2-groups. This solves Problem Nr. 1260 stated by Y.Berkovich in [1].
\end{abstract}

\section{INTRODUCTION AND KNOWN RESULTS}

We consider here only finite $p$-groups and our notation is standard. Conjugate subgroups have the same order and so it is of interest to consider the converse of this fact. This is done in Theorems 2.1 and 2.2.

If $H$ is a non-normal subgroup of a $p$-group $G$, then the normal closure $H^{G}$ of $H$ in $G$ is always a proper subgroup of $G$. Therefore, the largest possibility for $H^{G}$ is that $H^{G}$ is a maximal subgroup of $G$. We determine

2000 Mathematics Subject Classification. $20 \mathrm{D} 15$.

Key words and phrases. Minimal nonabelian p-groups, metacyclic p-groups, 2-groups of maximal class, central products, Hamiltonian 2-groups. 
in Theorem 2.3 all such $p$-groups $G$ with $\left|G: H^{G}\right|=p$ for each non-normal subgroup $H$ of $G$.

If a 2-group $G$ has only one conjugate class of four-subgroups, then $G$ is either semidihedral or $G$ has exactly three involutions and such groups are classified in [6]. Here we consider the next case, where a 2-group has exactly two conjugate classes of four-subgroups and prove Theorem 2.4.

In the proof of our theorems we shall use the following known results.

Proposition 1.1 (O. Taussky, see [3, Lemma 1.1(s)]). If $G$ is a nonabelian 2-group with $\left|G: G^{\prime}\right|=4$, then $G$ is of maximal class and so $G$ is dihedral, semidihedral or generalized quaternion.

Proposition 1.2 (N.Blackburn, see [2, Theorem 2]). The following conditions for a nonabelian p-group $G$ are equivalent:

(a) $G$ is metacyclic.

(b) The quotient group $G / R$ is metacyclic for some $G$-invariant subgroup $R$ of index $p$ in $G^{\prime}$.

Proposition 1.3 (L.Redei, see [3, Lemma 3.1]). Let $G$ be a minimal nonabelian p-group. Then $G=\langle a, b\rangle,\left|G^{\prime}\right|=p, \Phi(G)=Z(G)$ and one of the following holds:

(a) $a^{p^{m}}=b^{p^{n}}=c^{p}=1,[a, b]=c,[a, c]=[b, c]=1, m \geq n \geq 1$, where $|G|=p^{m+n+1}$ and in case $p=2$ we have $m>1$. In this case $G$ is non-metacyclic, $\left|\Omega_{1}(G)\right|=p^{3}$ and $G^{\prime}$ is a maximal cyclic subgroup of $G$.

(b) $a^{p^{m}}=b^{p^{n}}=1, a^{b}=a^{1+p^{m-1}}, m \geq 2, n \geq 1$, where $|G|=p^{m+n}$ and $G$ is metacyclic.

(c) $G \cong Q_{8}$ is quaternion.

Proposition 1.4 (B. Huppert [9, Satz 7.12]). Let $G$ be a p-group all of whose subgroups are normal in $G$. Then $G$ is either abelian or $p=2$ and $G$ is Hamiltonian, i.e., $G=Q \times V$ with $Q \cong Q_{8}$ and $V$ is elementary abelian.

Proposition 1.5. Let $G$ be a p-group with $\left|G^{\prime}\right|=p$. If $H$ is a minimal nonabelian subgroup of $G$, then $G=H C_{G}(H)$.

Proof. Set $H=\left\langle x_{1}, x_{2}\right\rangle$ and $C_{i}=C_{G}\left(x_{i}\right), i=1,2$, so that $\left|G: C_{i}\right|=p$. Considering $C=C_{1} \cap C_{2}$, we have $C=C_{G}(H), H \cap C=Z(H)$ and $|G: C| \leq$ $p^{2}$. Therefore, $|H C|=(|H||C|):|H \cap C|=p^{2}|C| \geq|G|$ which gives $G=H C$.

Proposition 1.6 ([8, Corollary 2.4]). Let $G$ be a nonabelian 2-group all of whose minimal nonabelian subgroups are isomorphic to $Q_{8}$. Then $G=$ $Q \times V$, where $Q \cong Q_{2^{n}}$ is a generalized quaternion group of order $2^{n}, n \geq 3$, and $V$ is elementary abelian. 
Lemma 1.7. Let $G$ be a minimal nonabelian p-group which possesses nonnormal subgroups (i.e., $G$ is distinct from $Q_{8}$ ) and such that any two nonnormal subgroups of the same order are conjugate in $G$. Then $G$ is isomorphic to $M_{p^{n}}$.

Proof. (Y.Berkovich) Note that every non-normal subgroup of $G$ has exactly $p$ conjugates. Therefore, $G$ cannot contain $>p$ non-normal subgroups of the same order. If $|G|=p^{3}$, then $G \cong M_{p^{3}}, p>2$, and so we may assume that $|G|>p^{3}$.

Suppose that $\Omega_{1}(G) \cong E_{p^{3}}$. If $\Omega_{1}(G) \not \leq Z(G)$, then $\Omega_{1}(G)$ has exactly $p^{2}$ noncentral subgroups of order $p$, a contradiction. Suppose that $\Omega_{1}(G) \leq Z(G)$ so that $G$ is a group given in Proposition 1.3(a) with $n>1$. The subgroup $H=\left\langle a, \Omega_{1}(G)\right\rangle$ is abelian of type $\left(p^{m}, p, p\right)$ and no cyclic subgroup $Y$ of order $p^{m},(m \geq 2)$ in $H$ is normal in $G$ (otherwise, the fact that $Y$ is not central in $G$ would imply that $G^{\prime}=\langle c\rangle<Y$, contrary to the fact that $G^{\prime}$ is a maximal cyclic subgroup of $G$ ). There are exactly $p^{2}$ cyclic subgroups of order $p^{m}$ in $H$, a contradiction.

Thus, $\Omega_{1}(G) \cong E_{p^{2}}$ so that $G$ is a group given in Proposition 1.3(b). If $n=1$, then $G \cong M_{p^{s}}$. Next we assume that $n \geq 2$. If $n \geq m$, then $\langle b\rangle$ and $\langle b a\rangle$ are of order $p^{n}$ and they are not $\langle a\rangle$-invariant. Since $\langle b\rangle^{G}=\langle b\rangle \times G^{\prime}$ and $\langle b a\rangle \not \leq\langle b\rangle \times G^{\prime},\langle b\rangle$ and $\langle b a\rangle$ are not conjugate in $G$, a contradiction. Thus, $n<m$. Let $y \in\langle a\rangle$ be of order $o(b)=p^{n}$. Then $\langle b\rangle$ and $\langle b y\rangle$ are of order $p^{n}$, they are not $\langle a\rangle$-invariant and they are not $G$-conjugate, a contradiction.

LEMMA 1.8. Let $G$ be a minimal nonabelian p-group which possesses nonnormal cyclic subgroups and such that any two non-normal cyclic subgroups are conjugate in $G$. Then $G$ is isomorphic to $M_{p^{n}}$.

Proof. Note that the conjugate class of non-normal cyclic subgroups is of size $p$. If $|G|=p^{3}$, then $G \cong M_{p^{3}}, p>2$, and so we may assume that $|G|>p^{3}$.

Suppose that $\Omega_{1}(G) \cong E_{p^{3}}$. If $\Omega_{1}(G) \not \leq Z(G)$, then $\Omega_{1}(G)$ has exactly $p^{2}$ noncentral subgroups of order $p$, a contradiction. Suppose that $\Omega_{1}(G) \leq Z(G)$ so that $G$ is a group given in Proposition 1.3(a) with $n>1$. The subgroup $H=\left\langle a, \Omega_{1}(G)\right\rangle$ is abelian of type $\left(p^{m}, p, p\right)$ and no cyclic subgroup $Y$ of order $p^{m},(m \geq 2)$ in $H$ is normal in $G$ (otherwise, the fact that $Y$ is not central in $G$ would imply that $G^{\prime}=\langle c\rangle<Y$, contrary to the fact that $G^{\prime}$ is a maximal cyclic subgroup of $G$ ). There are exactly $p^{2}$ cyclic subgroups of order $p^{m}$ in $H$, a contradiction.

Thus, $\Omega_{1}(G) \cong E_{p^{2}}$ so that $G$ is a group given in Proposition 1.3(b). If $n=1$, then $G \cong M_{p^{s}}$. Next we assume that $n \geq 2$. If $n \geq m$, then $\langle b\rangle$ and $\langle b a\rangle$ are of order $p^{n}$ and they are not $\langle a\rangle$-invariant. Since $\langle b\rangle^{G}=\langle b\rangle \times G^{\prime}$ and $\langle b a\rangle \not \leq\langle b\rangle \times G^{\prime},\langle b\rangle$ and $\langle b a\rangle$ are not conjugate in $G$, a contradiction. Thus, 
$n<m$. Let $y \in\langle a\rangle$ be of order $o(b)=p^{n}$. Then $\langle b\rangle$ and $\langle b y\rangle$ are of order $p^{n}$, they are not $\langle a\rangle$-invariant and they are not $G$-conjugate, a contradiction.

Proposition 1.9 (Janko [7]). Let $G$ be a finite p-group which possesses non-normal subgroups and we assume that each non-normal subgroup of $G$ is contained in exactly one maximal subgroup. Then one of the following holds:

(a) $G$ is minimal nonabelian;

(b) $G$ is a 2-group of maximal class;

(c) $G=\langle a, b\rangle$ is a non-metacyclic 2-group, where $a^{2^{n}}=1, n \geq 3$, o(b) $=$ 2 or $4, a^{b}=a k, k^{2}=a^{-4},[k, a]=1, k^{b}=k^{-1}$ and we have either:

(c1) $b^{2} \in\left\langle a^{2^{n-1}}, a^{2} k\right\rangle \cong E_{4}$, in which case $|G|=2^{n+2}, \Phi(G)=$ $\left\langle a^{2}\right\rangle \times\left\langle a^{2} k\right\rangle \cong C_{2^{n-1}} \times C_{2}, Z(G)=\left\langle a^{2^{n-1}}\right\rangle \times\left\langle a^{2} k\right\rangle \cong E_{4}$, or

(c2) $b^{2} \notin\left\langle a^{2^{n-1}}, a^{2} k\right\rangle \cong E_{4}$, in which case $o(b)=4,|G|=2^{n+3}$, $\Phi(G)=\left\langle a^{2}\right\rangle \times\left\langle a^{2} k\right\rangle \times\left\langle b^{2}\right\rangle \cong C_{2^{n-1}} \times C_{2} \times C_{2}, Z(G)=\left\langle a^{2^{n-1}}\right\rangle \times$ $\left\langle a^{2} k\right\rangle \times\left\langle b^{2}\right\rangle \cong E_{8}$.

(d) $G=\langle a, b\rangle$ is a splitting metacyclic 2-group, where $a^{2^{n}}=b^{4}=1$, $n \geq 3, a^{b}=a^{-1} z^{\epsilon}, \epsilon=0,1, z=a^{2^{n-1}}$. Here $|G|=2^{n+2}, \Phi(G)=$ $\left\langle a^{2}\right\rangle \times\left\langle b^{2}\right\rangle \cong C_{2^{n-1}} \times C_{2}, Z(G)=\langle z\rangle \times\left\langle b^{2}\right\rangle \cong E_{4}, G^{\prime}=\left\langle a^{2}\right\rangle \cong C_{2^{n-1}}$. Since a centralizes $\Phi(G)$ and $b$ inverts each element of $\Phi(G)$, it follows that each subgroup of $\Phi(G)$ is normal in $G$.

Proposition 1.10 ([2, Lemma 3.1]). Let $G$ be a minimal nonabelian $p$ group. Then $d(G)=2,\left|G^{\prime}\right|=p$, and $Z(G)=\Phi(G)$. If $|G|>p^{3}$, then $G$ is non-metacyclic if and only if $\Omega_{1}(G) \cong E_{p^{3}}$ is elementary abelian of order $p^{3}$.

Proposition 1.11 ([4, Theorem 1.1]). Let $G$ be a nonabelian 2-group containing an involution $t$ such that $C_{G}(t)=\langle t\rangle \times C$, where $C \cong C_{2^{m}}, m \geq 2$. Assume in addition that $G$ has no elementary abelian subgroup of order 8 and $t \notin \Phi(G)$. Then either $G \cong M_{2^{s}}, s \geq 4$, (a 2-group of order $2^{s}$ and class 2 which has a cyclic subgroup of index 2$)$ or $G$ has a subgroup $S$ of index $\leq 2, S=A L$, where $A \cong C_{2^{m}}, m \geq 2, L=\langle b, t| b^{2^{n-1}}=t^{2}=1, b^{t}=$ $\left.b^{-1}, n \geq 3\right\rangle \cong D_{2^{n}}$ is normal in $G, A \cap L=Z(L),[A, t]=1, C_{G}(t)=\langle t\rangle \times A$, $\Omega_{1}(G)=\Omega_{1}(S)=\Omega_{2}(A) * L$ (a central product). If $|G: S|=2$, then there is an element $x \in G-S$ such that $t^{x}=t b$.

Proposition 1.12 ([5, Theorem 3.1]). Let $G$ be a 2-group containing an involution $t$ such that $C_{G}(t)=\langle t\rangle \times Q$, where $Q$ is a generalized quaternion group of order $2^{m}, m \geq 3$. We assume in addition that $G$ possesses a normal four-subgroup $U$ such that $t \notin U$. Then $G$ has a normal subgroup $S$ containing $U C_{G}(t)$ such that $|G / S| \leq 4$. If $G$ acts transitively on the set of involutions in $S-U$, then $|G / S|=4$ and $S \cong Q_{2^{m}} * D_{2^{m}}$ with $Q_{2^{m}} \cap D_{2^{m}}=Z\left(D_{2^{m}}\right)$. 


\section{NeW RESUlts}

THEOREM 2.1. Let $G$ be a finite p-group which possesses non-normal subgroups and such that any two non-normal subgroups of the same order are conjugate in $G$. Then $G$ is isomorphic to $M_{p^{n}}$.

Proof. Let $G$ be a $p$-group of the smallest possible order which possesses non-normal subgroups and such that any two non-normal subgroups of the same order are conjugate in $G$ but $G$ is not isomorphic to any group $M_{p^{n}}$. Let $R$ be a subgroup of order $p$ in $G^{\prime} \cap Z(G)$. Then either $G / R \cong M_{p^{n}}$ or $G / R$ is Dedekindian in which case $G / R$ is either abelian or $p=2$ and $G / R$ is Hamiltonian (Proposition 1.4).

(i) Suppose that $G / R \cong M_{p^{n}}$. Let $H / R$ be a cyclic subgroup of index $p$ in $G / R$ so that $R<G^{\prime}<H$ and $G^{\prime} / R \cong C_{p}$. Since $G / R$ is metacyclic, Proposition 1.2 gives that $G$ is also metacyclic. In that case $G^{\prime} \cong C_{p^{2}}$ is cyclic of order $p^{2}$ which implies that $H$ is cyclic. Hence $G$ has a cyclic subgroup of index $p$, but such groups cannot have a factor-group isomorphic to $M_{p^{n}}$.

(ii) Suppose that $G / R$ is abelian so that $G^{\prime}=R$ is of order $p$. If $G$ is minimal nonabelian, then Lemma 1.7 implies that $G \cong M_{p^{n}}$, a contradiction. If each minimal nonabelian subgroup of $G$ is quaternion, then Proposition 1.6 implies that $G$ is Hamiltonian, a contradiction. It follows that $G$ possesses a minimal nonabelian subgroup $H \neq G$ such that $H \neq Q_{8}$. By Proposition 1.5, $G=H C_{G}(H)$ and so $H$ satisfies the assumptions of Lemma 1.7 which gives that

$$
H=\left\langle a, b \mid a^{p^{n-1}}=b^{p}=1, n \geq 3, a^{b}=a r, r=a^{p^{n-2}}\right\rangle \cong M_{p^{n}},
$$

where in case $p=2$, we have $n \geq 4$. Set $a^{p}=s$ so that $H^{\prime}=\langle r\rangle \leq\langle s\rangle=$ $Z(H)=\Phi(H) \leq Z(G)$. Let $Y$ be a subgroup of $C_{G}(H)$ which contains $\langle s\rangle$ as a subgroup of index $p$ so that $Y$ is abelian. Note that $p$ non-central subgroups $\left\langle b r^{i}\right\rangle, i=0,1, \ldots, p-1$ of order $p$ in $H$ form a complete conjugate class in $G$. It follows that each element of order $p$ in $G-H$ must be central in $G$. Suppose that there is an element $y \in Y-\langle s\rangle$ of order $p$. Then $y$ is central in $G$. Since $o(b y)=p$ and $b y \notin H$, by must be central in $G$. But then $b$ would be also central in $G$, a contradiction. We have proved that $Y$ is cyclic. Choose an element $l \in Y-\langle s\rangle$ such that $l^{p}=s^{-1}$. But then $a l \notin H$, $(a l)^{p}=a^{p} l^{p}=s s^{-1}=1$ and so al must be central in $G$. On the other hand, $(a l)^{b}=(a r) l=(a l) r$ and so $a l \notin Z(G)$, a contradiction.

(iii) We have proved that $p=2$ and $G / R$ must be Hamiltonian for any subgroup $R$ of order 2 which is contained in $G^{\prime} \cap Z(G)$. It follows (Proposition 1.4) that $G=Q F$ with normal subgroups $Q$ and $F$, where $Q \cap F=R, Q / R \cong$ $Q_{8}, R<G^{\prime}=\Phi(G)<Q,\left|G^{\prime}\right|=4, Q^{\prime}$ covers $G^{\prime} / R$ and $F / R$ is elementary abelian. If $Q^{\prime}=G^{\prime}$, then Proposition 1.1 implies that $Q$ is of maximal class and therefore $Q / R \cong D_{8}$, a contradiction. Thus, $G^{\prime}=Q^{\prime} \times R \cong E_{4}$ and $G^{\prime} \leq Z(G)$ and $G / X$ is Hamiltonian for each subgroup $X$ of order 2 in 
$G^{\prime}=\Phi(G)$. Suppose that $G$ possesses a minimal nonabelian subgroup $M$ which is not isomorphic to $Q_{8}$. By the previous sentence, $M>G^{\prime}$ and since $G^{\prime} \leq Z(M)=\Phi(M)$, we have $\Phi(M)=\Phi(G)=G^{\prime}$ and $|M|=16$. Let $X_{0}$ be a subgroup of order 2 in $G^{\prime}$ with $X_{0} \neq M^{\prime}$. Then $M / X_{0} \cong Q_{8}$ (noting that $G\left(X_{0}\right.$ is Hamiltonian) and so $\Omega_{1}(M)=G^{\prime} \cong E_{4}$ and $\exp (M)=4$. By Proposition 1.3, $M=\left\langle a, b \mid a^{4}=b^{4}=1, a^{b}=a^{-1}\right\rangle$ with $Z(M)=\Phi(M)=$ $\left\langle a^{2}, b^{2}\right\rangle=G^{\prime}$. But then $M /\left\langle b^{2}\right\rangle \cong D_{8}$, contrary to the fact that $G /\left\langle b^{2}\right\rangle$ is Hamiltonian. We have proved that each minimal nonabelian subgroup of $G$ is quaternion. But then, by Proposition 1.6, $G^{\prime}$ is cyclic, a contradiction. Our theorem is proved.

By using Lemma 1.8, we prove in exactly the same way (with minor changes):

TheOREM 2.2. Let $G$ be a finite $p$-group which possesses non-normal cyclic subgroups and such that any two non-normal cyclic subgroups are conjugate in $G$. Then $G$ is isomorphic to $M_{p^{n}}$.

Proof. Let $G$ be a $p$-group of the smallest possible order which possesses exactly one conjugate class of cyclic non-normal subgroups but $G$ is not isomorphic to any group $M_{p^{n}}$. Let $R$ be a subgroup of order $p$ in $G^{\prime} \cap Z(G)$. Note that our hypothesis is inherited by factor-groups. Indeed, let $F / R$ and $H / R$ be two non-normal cyclic subgroups in $G / R$. Let $F_{1}$ and $H_{1}$ be cyclic subgroups in $F$ and $H$ which cover $F / R$ and $H / R$, respectively. Since $F_{1}$ and $H_{1}$ are non-normal, they are conjugate in $G$ and so $F / R$ and $H / R$ are conjugate in $G / R$. Then either $G / R \cong M_{p^{n}}$ or $G / R$ is Dedekindian in which case $G / R$ is either abelian or $p=2$ and $G / R$ is Hamiltonian (Proposition 1.4).

(i) Suppose that $G / R \cong M_{p^{n}}$. Let $H / R$ be a cyclic subgroup of index $p$ in $G / R$ so that $R<G^{\prime}<H$ and $G^{\prime} / R \cong C_{p}$. Since $G / R$ is metacyclic, Proposition 1.2 gives that $G$ is also metacyclic. In that case $G^{\prime} \cong C_{p^{2}}$ is cyclic of order $p^{2}$ which implies that $H$ is cyclic. Hence $G$ has a cyclic subgroup of index $p$, but such groups cannot have a factor-group isomorphic to $M_{p^{n}}$.

(ii) Suppose that $G / R$ is abelian so that $G^{\prime}=R$ is of order $p$. If $G$ is minimal nonabelian, then Lemma 1.8 implies that $G \cong M_{p^{n}}$, a contradiction. If each minimal nonabelian subgroup of $G$ is quaternion, then Proposition 1.6 implies that $G$ is Hamiltonian, a contradiction. It follows that $G$ possesses a minimal nonabelian subgroup $H \neq G$ such that $H \neq Q_{8}$. By Proposition 1.5, $G=H C_{G}(H)$ and so $H$ satisfies the assumptions of Lemma 1.8 which gives that

$$
H=\left\langle a, b \mid a^{p^{n-1}}=b^{p}=1, n \geq 3, a^{b}=a r, r=a^{p^{n-2}}\right\rangle \cong M_{p^{n}},
$$

where in case $p=2$, we have $n \geq 4$. Set $a^{p}=s$ so that $H^{\prime}=\langle r\rangle \leq\langle s\rangle=$ $Z(H)=\Phi(H) \leq Z(G)$. Let $Y$ be a subgroup of $C_{G}(H)$ which contains $\langle s\rangle$ as a subgroup of index $p$ so that $Y$ is abelian. Note that $p$ non-central 
subgroups $\left\langle b r^{i}\right\rangle, i=0,1, \ldots, p-1$ of order $p$ in $H$ form a complete conjugate class in $G$. It follows that each element of order $p$ in $G-H$ must be central in $G$. Suppose that there is an element $y \in Y-\langle s\rangle$ of order $p$. Then $y$ is central in $G$. Since $o(b y)=p$ and $b y \notin H$, by must be central in $G$. But then $b$ would be also central in $G$, a contradiction. We have proved that $Y$ is cyclic. Choose an element $l \in Y-\langle s\rangle$ such that $l^{p}=s^{-1}$. But then al $\notin H$, $(a l)^{p}=a^{p} l^{p}=s s^{-1}=1$ and so $a l$ must be central in $G$. On the other hand, $(a l)^{b}=(a r) l=(a l) r$ and so $a l \notin Z(G)$, a contradiction.

(iii) We have proved that $p=2$ and $G / R$ must be Hamiltonian for any subgroup $R$ of order 2 which is contained in $G^{\prime} \cap Z(G)$. It follows (Proposition 1.4) that $G=Q F$ with normal subgroups $Q$ and $F$, where $Q \cap F=R, Q / R \cong$ $Q_{8}, R<G^{\prime}=\Phi(G)<Q,\left|G^{\prime}\right|=4, Q^{\prime}$ covers $G^{\prime} / R$ and $F / R$ is elementary abelian. If $Q^{\prime}=G^{\prime}$, then Proposition 1.1 implies that $Q$ is of maximal class and therefore $Q / R \cong D_{8}$, a contradiction. Thus, $G^{\prime}=Q^{\prime} \times R \cong E_{4}$ and $G^{\prime} \leq Z(G)$ and $G / X$ is Hamiltonian for each subgroup $X$ of order 2 in $G^{\prime}=\Phi(G)$. Suppose that $G$ possesses a minimal nonabelian subgroup $M$ which is not isomorphic to $Q_{8}$. By the previous sentence, $M>G^{\prime}$ and since $G^{\prime} \leq Z(M)=\Phi(M)$, we have $\Phi(M)=\Phi(G)=G^{\prime}$ and $|M|=16$. Let $X_{0}$ be a subgroup of order 2 in $G^{\prime}$ with $X_{0} \neq M^{\prime}$. Then $M / X_{0} \cong Q_{8}$ (noting that $G / X_{0}$ is Hamiltonian) and so $\Omega_{1}(M)=G^{\prime} \cong E_{4}$ and $\exp (M)=4$. By Proposition 1.3, $M=\left\langle a, b \mid a^{4}=b^{4}=1, a^{b}=a^{-1}\right\rangle$ with $Z(M)=\Phi(M)=$ $\left\langle a^{2}, b^{2}\right\rangle=G^{\prime}$. But then $M /\left\langle b^{2}\right\rangle \cong D_{8}$, contrary to the fact that $G /\left\langle b^{2}\right\rangle$ is Hamiltonian. We have proved that each minimal nonabelian subgroup of $G$ is quaternion. But then, by Proposition 1.6, $G^{\prime}$ is cyclic, a contradiction. Our theorem is proved.

THEOREM 2.3. Let $G$ be a finite p-group which possesses non-normal subgroups and such that $\left|G: H^{G}\right|=p$ for each non-normal subgroup $H$ of $G$. Then $G$ is either the nonabelian group of order $p^{3}, p>2$, and exponent $p$ or $G$ is one of the following metacyclic p-groups:

(i) $G=\left\langle a, b \mid a^{p^{2}}=b^{p^{n}}=1, n=1,2, a^{b}=a^{1+p}\right\rangle$, where $G$ is minimal nonabelian of order $p^{n+2}$.

(ii) $G$ is a 2-group of maximal class distinct from the quaternion group $Q_{8}$.

(iii) $G=\left\langle a, b \mid a^{2^{n}}=b^{4}=1, n \geq 3, a^{b}=a^{-1} z^{\epsilon}, \epsilon=0,1, z=a^{2^{n-1}}\right\rangle$, where $G$ is a splitting metacyclic group of order $2^{n+2}, n \geq 3, \Phi(G)=\left\langle a^{2}\right\rangle \times$ $\left\langle b^{2}\right\rangle \cong C_{2^{n-1}} \times C_{2}, Z(G)=\langle z\rangle \times\left\langle b^{2}\right\rangle \cong E_{4}$ and $G^{\prime}=\left\langle a^{2}\right\rangle \cong C_{2^{n-1}}$.

Conversely, all the above groups satisfy the assumptions of our theorem.

Proof. Let $H$ be a non-normal subgroup of a $p$-group $G$ satisfying the assumptions of Theorem 2.3. Then $H$ is contained in exactly one maximal subgroup of $G$. It follows that $G$ satisfies the assumptions of Proposition 1.9 and so $G$ must be isomorphic to some of the groups stated in that proposition. 
(a) Let $G$ be a minimal nonabelian group distinct from $Q_{8}$ (since $Q_{8}$ has no non-normal subgroups). We use Proposition 1.10 and see that $Z(G)=$ $\Phi(G)$ with $|G: \Phi(G)|=p^{2}$ and $\left|G^{\prime}\right|=p$. Let $s$ be an element of order $p$ in $G-\Phi(G)$. Then $\langle s\rangle$ is not normal in $G$ and $\langle s\rangle^{G}=\left\langle s, G^{\prime}\right\rangle \cong E_{p^{2}}$ which implies that $G$ is nonabelian of order $p^{3}$.

In what follows we assume that there are no elements of order $p$ in $G-$ $\Phi(G)$. Let $b \in G-\Phi(G)$ be such that $\langle b\rangle$ is not normal in $G$ which implies that $G^{\prime} \not\left\langle\langle b\rangle\right.$. Since $\langle b\rangle^{G}=\left\langle b, G^{\prime}\right\rangle$, it follows that $\Phi(G)=\left\langle b^{p}\right\rangle \times G^{\prime},|G|>p^{3}$, and $\Omega_{1}(G)=\Omega_{1}(\Phi(G)) \cong E_{p^{2}}$. By Proposition 1.10, $G$ is metacyclic and so there is $a \in G-\langle b, \Phi(G)\rangle$ such that $\left\langle a^{p}\right\rangle=G^{\prime}$ and we may set $a^{b}=a^{1+p}$ and $o(b)=p^{n}, n \geq 2$. Assume that $n>2$ and let $x$ be an element of order $p^{2}$ in $\langle b\rangle$ so that $H=\langle a x\rangle$ is not normal in $G$ since $(a x)^{b}=(a x) a^{p} \notin H$. But $H^{G}=H \times G^{\prime}$ and $\left|G: H^{G}\right| \geq p^{2}$, a contradiction. We have obtained the groups stated in part (i) of our theorem.

(b) Suppose that $G$ is a 2-group of maximal class distinct from $Q_{8}$. Let $\langle a\rangle$ be the unique cyclic subgroup of index 2 in $G$ and set $o(a)=2^{n}, n \geq 2$, so that $|G|=2^{n+1}$. Let $X$ be a proper normal subgroup of $G$ with $X \not \leq\langle a\rangle$. Set $z=a^{2^{n-1}}$ so that $X \geq\langle z\rangle=Z(G)$ and let $y$ be an element in $X-\langle a\rangle$. We have $y^{2} \in\langle z\rangle$ and $C_{G}(y)=\langle y, z\rangle$. It follows that all $2^{n-1}$ conjugates of $y$ in $G$ are contained in $X-\langle a\rangle$ and so $|X|>2^{n-1}$ which implies that $|X|=2^{n}$ and so $X$ is a maximal subgroup of $G$. We see that each 2-group of maximal class distinct from $Q_{8}$ satisfies the assumptions of our theorem.

(c) Suppose that $G$ is a non-metacyclic 2-group which is isomorphic to one of the groups in part (c) of Proposition 1.9. We have $a^{b}=a k$ and so the abelian subgroup $\langle a, k\rangle$ of order $2^{n+1}$ (containing $\langle k\rangle=G^{\prime}$ ) is normal in $G$ which implies that $\langle a\rangle^{G}=\langle a, k\rangle$. Since in case (c2) of Proposition 1.9 we have $|G:\langle a, k\rangle|=4$, it follows that we must be in case (c1) of that proposition, where $b^{2} \in\left\langle a^{2^{n-1}}, a^{2} k\right\rangle \cong E_{4}$. From $a^{b}=a k$ follows $b^{a}=k b$ and so $\langle b\rangle^{G}=\langle b, k\rangle$, where $k^{b}=k^{-1}$. By our assumption, $\left|G:\langle b\rangle^{G}\right|=2$ and so $b^{2} \notin\langle a\rangle$ which forces that either $b^{2}=a^{2} k$ or $b^{2}=a^{2} k \cdot a^{2^{n-1}}$. We have $(a b)^{a}=(a b) k^{-1}$ and so $\langle a b\rangle^{G}=\langle a b, k\rangle$, where $k^{a b}=k^{-1}$ and

$$
(a b)^{2}=a b a b=a b^{2} a^{b}=a b^{2} a k=b^{2}\left(a^{2} k\right) .
$$

By our assumption, $|G:\langle a b, k\rangle|=2$ and so we must have $(a b)^{2} \notin\langle k\rangle$. If $b^{2}=$ $a^{2} k$, then $(a b)^{2}=1$, a contradiction. If $b^{2}=\left(a^{2} k\right) a^{2^{n-1}}$, then $(a b)^{2}=a^{2^{n-1}}$ which gives $(a b)^{2} \in\langle k\rangle$ since $k^{2}=a^{-4}$ and $a^{2^{n-1}} \in\left\langle a^{-4}\right\rangle$. Hence $G$ cannot be isomorphic to any group in part (c) of Proposition 1.9.

(d) Suppose that $G$ is a metacyclic 2-group given in part (d) of Proposition 1.9. Then we see easily that these groups satisfy the assumptions of our theorem which gives the groups stated in part (iii) of our theorem.

TheOREM 2.4. Let $G$ be a finite 2-group which possesses exactly two conjugate classes of four-subgroups. Then $G$ must be one of the following groups: 
(i) $G \cong D_{2^{n}}$, a dihedral group of order $2^{n}, n \geq 3$.

(ii) $G$ has a subgroup $S$ of index $2, S=A L$, where $A \cong C_{2^{m}}, m \geq 2$, is cyclic of order $2^{m}, L=\left\langle b, t \mid b^{2^{n-1}}=t^{2}=1, b^{t}=b^{-1}, n \geq 3\right\rangle \cong D_{2^{n}}$ is dihedral of order $2^{n}, L$ is normal in $G, A \cap L=Z(L),[A, t]=1$, $C_{G}(t)=\langle t\rangle \times A, \Omega_{1}(G)=\Omega_{1}(S)=\Omega_{2}(A) * L$ (a central product), and there is an element $x \in G-S$ such that $t^{x}=t b$.

(iii) $G$ has a normal subgroup $S$ of index 4 , where $S \cong Q_{2^{m}} * D_{2^{m}}, m \geq 3$, is a central product of a generalized quaternion group of order $2^{m}$ with a dihedral group of order $2^{m}, Q_{2^{m}} \cap D_{2^{m}}=Z\left(D_{2^{m}}\right), \Omega_{1}(G)=S$ and if $t$ is a non-central involution in $D_{2^{m}}$, then $C_{G}(t)=\langle t\rangle \times Q_{2^{m}}$.

Proof. Let $G$ be a 2 -group which possesses exactly two conjugate classes of four-subgroups. If $G$ has no normal four-subgroup, then $G$ is either cyclic or a 2-group of maximal class distinct from $D_{8}$. It follows that $G \cong D_{2^{n}}$, $n \geq 4$. In what follows we assume that $G$ has a normal four-subgroup $U$ and also we may assume that $G$ is not isomorphic to $D_{8}$.

Suppose that $U \leq Z(G)$ and set $U=\left\langle u_{1}, u_{2}\right\rangle$. Let $t$ be an involution in $G-U$ so that $V=\left\langle t, u_{1}\right\rangle$ is a four-subgroup which is not conjugate to $U$. Also, the four-subgroup $W=\left\langle t, u_{2}\right\rangle$ is not conjugate to $U$. We have $V \cap U=\left\langle u_{1}\right\rangle$ and $W \cap U=\left\langle u_{2}\right\rangle$. Since $V$ must be conjugate in $G$ to $W$, it follows that $u_{1}$ and $u_{2}$ must be conjugate in $G$, a contradiction.

We have proved that $U \not Z Z(G)$ and we set $M=C_{G}(U)$ so that $|G: M|=2$. We set also $U=\langle u, z\rangle$, where $\langle z\rangle=U \cap Z(G)$. It is easy to see that $\Omega_{1}(M)=U$ so that $G$ has no elementary abelian subgroups of order 8. Indeed, let $t^{\prime}$ be an involution in $M-U$ and consider the four-subgroups $\left\langle t^{\prime}, u\right\rangle$ and $\left\langle t^{\prime}, z\right\rangle$. Since they must be conjugate in $G$, it follows that also $u$ and $z$ must be conjugate in $G$, a contradiction.

Let $t$ be a fixed involution in $G-M$. Then $C_{G}(t)=\langle t\rangle \times C_{M}(t)$, where $\Omega_{1}\left(C_{M}(t)\right)=\langle z\rangle$ so that $C_{M}(t)$ is either cyclic of order $\geq 4$ or generalized quaternion. Indeed, if $C_{M}(t)=\langle z\rangle$, then, by a well known result of M. Suzuki, $G$ would be of maximal class, which was excluded. Since $t^{u}=t z$ and $G$ has exactly one conjugate class of four-subgroups distinct from $U$, it follows that there is exactly one conjugate class of involutions in $G-M$. Hence, also $G-U$ contains exactly one conjugate class of involutions.

Assume that $C=C_{G}(t)$ is cyclic of order $\geq 4$. We are in a position to use Proposition 1.11. If $G \cong M_{2^{s}}, s \geq 4$, then $\Omega_{1}(G) \cong E_{4}$, a contradiction. Hence we must have the second possibility of that proposition. Here $|G: S|=$ 2 since all involutions in $L-\langle b\rangle$ must lie in a single conjugate class in $G$. We have obtained the groups stated in part (ii) of our theorem.

Assume that $Q=C_{G}(t) \cong Q_{2^{m}}, m \geq 3$, is a generalized quaternion group of order $2^{m}$. Since $t \notin U$, we are in a position to use Proposition 1.12. Note that $G$ must fuse all involutions in $G-U$ which gives us the groups stated in part (iii) of our theorem. 
ACKNOWLEDGEMENTS.

The author thanks Prof. Y. Berkovich for correcting an error in my proof of Theorem 2.3, part (i).

\section{REFERENCES}

[1] Y. Berkovich, Groups of prime power order, I and II (with Z. Janko), Walter de Gruyter, Berlin, 2008, to appear.

[2] Y. Berkovich, Short proofs of some basic characterization theorems of finite p-group theory, Glas. Mat. Ser. III 41(61) (2006), 239-258.

[3] Y. Berkovich and Z. Janko, Structure of finite p-groups with given subgroups, Contemporary Math. 402 (2006), 13-93.

[4] Z. Janko, Finite 2-groups with small centralizer of an involution, J. of Algebra 241 (2001), 818-826.

[5] Z. Janko, Finite 2-groups with small centralizer of an involution 2, J. of Algebra 245 (2001), 413-429.

[6] Z. Janko, A classification of finite 2-groups with exactly three involutions, J. of Algebra 291 (2005), 505-533.

[7] Z. Janko, Finite p-groups with a uniqueness condition for non-normal subgroups, Glas. Mat. Ser. III 40 (2007), 235-240.

[8] Z. Janko, On finite nonabelian 2-groups all of whose minimal nonabelian subgroups are of exponent 4, J. Algebra 315 (2007), 801-808.

[9] B. Huppert, Endliche Gruppen 1, Springer, Berlin, (1967).

\section{Z. Janko}

Mathematical Institute

University of Heidelberg

69120 Heidelberg

Germany

E-mail: janko@mathi.uni-heidelberg.de

Received: 19.9.2007.

Revised: 4.10.2007. 